\title{
A Comparative Study of Two Different Accommodative Intraocular Lenses: The Visual and Accommodative Amplitude Outcomes
}

\author{
(D) Hatice Nur Tarakçıoğlu¹, (D) Ulviye Yiğit¹, (D) Issmail Umut Onur1, (1) Abdullah Özkaya2,3 \\ 1istanbul Bakırköy Dr. Sadi Konuk Training and Research Hospital, Clinic of Ophtalmology, İstanbul, Turkey \\ 2istanbul Aydın University Faculty of Medicine, Department of Ophtalmology, istanbul, Turkey \\ ${ }^{3}$ Memorial Şişli Hospital, Clinic of Ophtalmology, Istanbul, Turkey
}

\section{Abstract}

Objective: To compare distance and near visual acuity along with accommodative amplitude in eyes implanted with the Tetraflex and the Tek-clear accommodating intraocular lenses (AIOLs).

Methods: Comparative, prospective, non-randomized study. Thirty-eight eyes of 26 patients implanted with the Tetraflex (17 eyes) and Tekclear (21 eyes) AIOLs were enrolled. Uncorrected distance visual acuity (UCDVA), best-corrected distance visual acuity (BCDVA), uncorrected near visual acuity (UCNVA), distance corrected near visual acuity (DCNVA), best-corrected near visual acuity (BCNVA) and spherical equivalent refraction were the parameters evaluated postoperatively. Also, the accommodative amplitude was assessed with subjective defocus method and objective anterior chamber depth measurement before and after the topical application of pilocarpine using a Scheimpflug-Placido disc topographer at postoperative month 3 and 6 .

Results: No statistically significant difference was found between the two AIOL types in regards to BCDVA, DCNVA, and BCNVA at months 1 , 3 , and $6(p>0.05)$. The mean UCDVA was significantly better in Tetraflex implanted eyes $(p=0.001, p=0.002, p=0.008)$, whereas the mean UCNVA was significantly better in Tek-clear implanted eyes $(p=0.008, p=0.01, p<0.0001)$ at postoperative month 1,3 , and 6 , respectively. Both subjective and objective accommodative amplitude assessments did not display a significant difference between the two groups at month 6 ( $p>0.05)$.

Conclusion: The Tetraflex accommodative IOL seemed to be better at UCDVA, whereas Tek-clear seemed better at UCNVA. The accommodation range of Tetraflex and Tek-clear lenses was comparable.

Keywords: Accommodation, cataract, intraocular lens, presbyopia

\section{INTRODUCTION}

Presbyopia is one of the most important problems in older patients with or without cataract (1-7). Spectacles, multifocal contact lenses, and several surgical treatment approaches were being used for the 'correction' of presbyopia (5-10). Presbyopia correction during cataract surgery is one of the important goals of anterior segment surgeons (5). Monovision correction, implantation of multifocal intraocular lenses (IOLS), and accommodative IOLS (AIOLS) are the most preferred choices for this purpose $(5,10)$. Monovision correction has the advantage of using cheaper IOLs with a relatively simple technique. However, leaving the eyes with faint anisometropia has many drawbacks such as diminished contrast sensitivity, risk of diplopia, and stereopsis problems $(5,10)$. On the other hand, multifocal IOLs are very popular among cataract surgeons with the advantage of simultaneous correction of far, intermediate, near vision, and also astigmatism with optional toric design $(5,6,9)$. However, reduced contrast sensitivity, halo, glare, and waxy vision are the major limitations of multifocal IOLs $(5,6,9)$. 
Alternatively, AIOLs are likely to mimic the accommodation reflex conceptually (5-8). There are three main types of AIOLs: single optic AIOLs, dual-optic AIOLs, and capsular bag refilling AIOLs $(7,8)$. Single optic designs work with accommodative effort, which is called as the focus-shift principle $(7,8)$. The lens optic of moves forward by contraction of the ciliary muscle, thereby increasing the refractive power of the IOL for better near vision $(7,8)$. Several brands of single optic AIOLs are available in the market, and several studies have been conducted to assess and compare the efficacy of AIOLs within the class and with other types of IOLs (8-10).

The Tetraflex AIOL (Lenstec Inc., FL, US) is a single-piece (optic), spherical optic, acrylic IOL that has been designed to utilize the two forces activated during accommodation-vitreous movement and ciliary swelling to ensure maximum forward movement for clear near vision (5-8). The Tek-clear AIOL (Tekia, Irvine, California, US) is also a single-piece (optic), hydrophilic acrylic IOL with symmetric optic and square edge design that has been approved for the treatment of presbyopia, which acts with a similar mechanism to Tetraflex AIOL (5-8). This study aimed to compare distance and near visual performances in the eyes of patients implanted with Tetraflex and Tek-clear single optic AIOLs during cataract surgery. Accommodative amplitudes of two AIOLs were also evaluated.

\section{METHODS}

This prospective, comparative, non-randomized study was conducted in accordance with the tenets of the Declaration of Helsinki, and approval was obtained from local ethics and review board. Ethics committee approval was obtained from the Ethics Committee of Bakırköy Dr. Sadi Konuk Training and Research Hospital (2011/117). Written informed consent was received from all of the included patients. The data of the patients who underwent cataract surgery with phacoemulsification and AIOL between December 2011 and April 2014 was recorded. The following criteria were required for inclusion: a) to be between the age of 40-65 years, b) to have unilateral or bilateral senile or presenile cataract, c) to have basic literacy and good cooperation, d) to commit the follow-up visits, e) to have the unwillingness to use spectacles postoperatively. The patients were not included if one or more of the following criteria were present: a) to have a concomitant ocular disease such as diabetic retinopathy, age-related macular degeneration, b) to have a history of previous laser treatment or intraocular surgery, c) to have spherical refractive error of \pm 6 diopters and above, d) to have a cylindrical refractive error of $\geq \pm 1.5$ diopters, e) to have signs for obsessive personality, f) to have jobs needing very concise near vision (watch repairer, jeweler, etc.), g) to show perioperative complications such as posterior capsule rupture, iris damage, irregular and large or small capsulorhexis.

\section{Preoperative Assessment}

The preoperative assessment involved a complete eye examination including uncorrected distance visual acuity (UCDVA) and best-corrected distance visual acuity (BCDVA), manifest refraction, keratometry (auto kerato-refractotonometer TRK-1P, Topcon, Tokyo, Japan), slit-lamp biomicroscopy, intraocular pressure (IOP) measurement, and dilated retinal examination. Biometry was obtained via an ultrasound biometer (Optikon, Roma, Italy). Immersion technique was preferred, and the required IOL power for emmetropia was calculated with the SRK-T formula. Distance visual acuity was measured via a projection chart from 4 meters and recorded in decimals. Near visual acuity was measured via a Turkish near vision chart, which was previously described (11). All examinations were performed by a single ophthalmologist (HNT).

\section{Surgical Technique}

All patients underwent standardized phacoemulsification and AIOL implantation performed by a single surgeon under local anesthesia. A $2.8 \mathrm{~mm}$ clear corneal incision was placed at the steepest corneal meridian. A continuous curvilinear capsulorhexis of 5-5.5 mm was created. Phacoemulsification was performed using the Infiniti Vision system (Alcon, Fort Worth, Texas, US). The choice of AIOL type did not depend on any special consideration because we had previously bought AIOLs in our institution, and patients were non-randomly allocated to one of the two AIOL types according to the availability of the required power. AIOLs were implanted into the capsular bag with a single-use IOL injector. None of the patients required corneal suturing because a leak-proof wound was obtained with hydration for all eyes. All patients received topical prednisolone acetate and ofloxacin five times a day following the surgery. Prednisolone acetate was tapered off after the first week and stopped after four weeks. Ofloxacin was stopped after two weeks postoperatively.

\section{Postoperative Assessment}

Postoperative examinations were performed at postoperative day 1 , week 1 , month 1 , month 3 , and month 6 . Each visit included assessment of visual acuity and manifest refraction, slit-lamp examination, and IOP measurement. In detail, UCDVA, BCDVA, uncorrected near visual acuity (UCNVA), distance-corrected near visual acuity (DCNVA), and best- 
corrected near visual acuity (BCNVA) were evaluated. Near visual acuity measurements were performed from 35 centimeters. Measurement of accommodative amplitude was performed at postoperative month 3 and 6 . Accommodative amplitude was evaluated with both subjective and objective methods. Defocus method was chosen as a subjective method, wherein minus lenses were used for stimulation of the accommodation. Under standard room illumination, the patient was seated with a full distance refractive correction while viewing the smallest letter on the visual acuity chart. Then, minus-power lenses were gradually increased in $0.25 \mathrm{D}$ steps until the visual target was blurred (minus-lenses-to-blur-method), and the added diopter was defined as the amplitude of accommodation (12). Anterior chamber depth was measured objectively with Sirius Scheimpflug-Placido topographer (Costruzione Strumenti Oftalmici, Florence, Italy) $(4,12)$. The distance between the anterior surface of the IOL and the corneal vertex was referred to as the anterior chamber depth and measured before and after pilocarpine instillation. Accommodative status was induced with two drops of pilocarpine $2 \%$ at 5 minutes interval, and the measurements were obtained after 30 minutes from the first instillation (13). Three consecutive measurements were taken and averaged before and after instillation of pilocarpine drops. The difference between the averages of two statuses, thus quantified drug-induced AIOL movement, which indirectly displayed the accommodation range objectively.

\section{Statistical Analysis}

All visual acuity values were converted to the logarithm of the minimum angle of resolution (LogMAR). Statistical analysis was performed using commercially available software (SPSS for Windows, version 20.0 SPSS Inc., Chicago, IL). Descriptive statistical results were described as the mean, standard deviation, and $95 \%$ confidence interval of the mean. The normality of the data was assessed using the Shapiro-Wilk test. According to the normality results, the Mann-Whitney $\mathrm{U}$ test or t-test was used for comparisons between groups or variables. The Wilcoxon test was used for repeated values. Chi-square and Fisher-exact test was used for the analysis of categorical variables. A p value of less than 0.05 was considered statistically significant.

\section{RESULTS}

A total of 39 eyes of 26 patients were initially included. One eye was then removed from the study because of posterior capsular rupture during phacoemulsification. Therefore, a total of 38 eyes were evaluated. The baseline parameters of $2 \mathrm{AIOL}$ groups were summarized in Table 1. Accordingly, two groups were homogenous concerning all parameters.

Seventeen eyes of 15 patients were implanted with Tetraflex AIOL, whereas 21 eyes of 19 patients were implanted with Tek-clear AIOL. Unilateral cataract surgery was performed in 14 patients (14 eyes), and bilateral surgery was performed in 12 patients (24 eyes). Of bilaterally operated patients, eight were implanted with Tetraflex/Tek-clear, two were implanted with Tetraflex/Tetraflex, and two were implanted with Tek-clear/Tek-clear AIOLs.

\subsection{Visual Outcomes}

Visual results were summarized in Table 2.

\subsubsection{Uncorrected distance visual acuity (UCDVA)}

The mean baseline UCDVA was not statistically different between the two groups $(p=0.8)$; however, the mean UCDVA was better in the Tetraflex group than that of the Tek-clear group at all followup visits (Table 2, Figure 1).

\subsubsection{Best-corrected distance visual acuity (BCDVA)}

The mean baseline BCDVA was not statistically different between the two groups $(p=0.8$ ). Also, the mean BCDVA was not statistically different between the two groups after that (Table 2).

Table 1. Baseline and demographic characteristics of the patients in Tetraflex and Tek-clear groups

\begin{tabular}{|l|l|l|l|}
\hline & Tetraflex & Tek-clear & $\mathbf{p}$ \\
\hline Age, years (range) & $55.9 \pm 7.9(48-65)$ & $52.3 \pm 7.9(45-58)$ & 0.2 \\
\hline Male/Female & $10 / 5$ & $9 / 10$ & 0.1 \\
\hline Right/Left & $7 / 10$ & $11 / 10$ & 0.4 \\
\hline IOL power, diopters (range) & $21.0 \pm 1.3(20.0-22.2)$ & $21.5 \pm 1.3(20.0-22.0)$ & 0.3 \\
\hline Axial length, mm (range) & $23.2 \pm 0.6(22.6-23.5)$ & $23.0 \pm 0.6(22.5-23.5)$ & 0.2 \\
\hline Mean Kavg, diopters (range) & $43.3 \pm 1.2(42.5-42.9)$ & $43.5 \pm 1.3(44.0-44.3)$ & 0.6 \\
\hline Mean Baseline UCDVA & $0.95 \pm 0.45$ LogMAR & $0.97 \pm 0.53$ LogMAR & 0.8 \\
\hline Mean Baseline BCDVA & $0.71 \pm 0.43$ LogMAR & $0.72 \pm 0.41$ LogMAR & 0.8 \\
\hline
\end{tabular}

IOL: Intraocular lens, mm: Millimeter, UCDVA: Uncorrected distance visual acuity, BCDVA: Best corrected distance visual acuity, $\mathrm{K}_{\mathrm{Avg}}$ : Average keratometry values, LogMAR: Logarithm of the minimum angle of resolution 


\subsubsection{Uncorrected near visual acuity (UCNVA)}

The mean UCNVA at month 1, 3 and 6 was better in the Tek-clear group than that of the Tetraflex group (Table 2, Figure 2). Also, at month $6,95.2 \%$ of the eyes in the Tek-clear group vs $94.1 \%$ of the eyes in the Tetraflex group had a UCNVA $\geq 20 / 80$ at month 6 $(p=0.001)$.

\subsubsection{Distance corrected near visual acuity (DCNVA)}

The mean DCNVA was not statistically different between the two groups at any of the follow-up visits (Table 2).

\subsubsection{Best-corrected near visual acuity (BCNVA)}

The mean BCNVA was not statistically different between the two groups at any of the follow-up visits (Table 2).

\begin{tabular}{|c|c|c|c|}
\hline & Tetraflex & Tek-clear & p \\
\hline \multicolumn{4}{|c|}{ UCDVA (Mean \pm SD) } \\
\hline Baseline & $0.95 \pm 0.45$ & $0.97 \pm 0.53$ & 0.825 \\
\hline Month 1 & $0.14 \pm 0.16$ & $0.34 \pm 0.19$ & $0.001 * *$ \\
\hline Month 3 & $0.12 \pm 0.15$ & $0.30 \pm 0.20$ & $0.002^{* *}$ \\
\hline Month 6 & $0.16 \pm 0.16$ & $0.30 \pm 0.21$ & $0.008^{* *}$ \\
\hline$p^{*}$ & $0.00001^{*}$ & $0.00001^{*}$ & \\
\hline \multicolumn{4}{|c|}{ BCDVA (Mean \pm SD) } \\
\hline Baseline & $0.71 \pm 0.43$ & $0.72 \pm 0.41$ & 0.813 \\
\hline Month 1 & $-0.01 \pm 0.05$ & $0.01 \pm 0.04$ & 0.161 \\
\hline Month 3 & $-0.01 \pm 0.05$ & $0.00 \pm 0.05$ & 0.713 \\
\hline Month 6 & $0.01 \pm 0.05$ & $0.00 \pm 0.04$ & 0.544 \\
\hline$p^{*}$ & $0.00001^{*}$ & $0.00001^{*}$ & \\
\hline \multicolumn{4}{|c|}{ UCNVA (Mean \pm SD) } \\
\hline Month 1 & $0.54 \pm 0.17$ & $0.41 \pm 0.16$ & $0.008^{* *}$ \\
\hline Month 3 & $0.49 \pm 0.16$ & $0.38 \pm 0.17$ & $0.011^{* *}$ \\
\hline Month 6 & $0.54 \pm 0.15$ & $0.36 \pm 0.16$ & $0.00001 * *$ \\
\hline \multicolumn{4}{|c|}{ DCNVA (Mean \pm SD) } \\
\hline Month 1 & $0.65 \pm 0.08$ & $0.67 \pm 0.07$ & 0.340 \\
\hline Month 3 & $0.59 \pm 0.09$ & $0.64 \pm 0.11$ & 0.144 \\
\hline Month 6 & $0.62 \pm 0.09$ & $0.63 \pm 0.11$ & 0.934 \\
\hline \multicolumn{4}{|c|}{ BCNVA (Mean \pm SD) } \\
\hline Month 1 & $0.05 \pm 0.05$ & $0.04 \pm 0.05$ & 0.798 \\
\hline Month 3 & $0.05 \pm 0.05$ & $0.04 \pm 0.05$ & 0.798 \\
\hline Month 6 & $0.08 \pm 0.07$ & $0.06 \pm 0.06$ & 0.284 \\
\hline \multicolumn{4}{|c|}{$\begin{array}{l}\text { UCDVA: Uncorrected distance visual acuity, BCDVA: Best corrected distance visual } \\
\text { acuity, UCNVA: Uncorrected near visual acuity, DCNVA: Distance-corrected near } \\
\text { visual acuity, BCNVA: Best corrected near visual acuity, SD: Standard deviation, *: } \\
\text { Represents the in-group p values which were statistically significant, }{ }^{* *} \text { : Represents } \\
\text { the inter-group p values which were statistically significant }\end{array}$} \\
\hline
\end{tabular}

\subsection{Manifest refraction}

There was a statistically significant difference in mean spherical equivalent refractive error between the Tek-clear and Tetraflex groups at all of the follow-up visits. The eyes in the Tek-clear group were more myopic than the eyes in the Tetraflex group (Table 3).

\subsection{The amplitude of accommodation (AA)}

There was not any statistically significant difference between the two groups in regards to the mean AA determined by subjective defocus method and objective measurement of mean change in anterior chamber depth with Scheimpflug topographer before and after topical pilocarpine (Table 3).

\subsection{Subjective patient satisfaction}

Patients were simply asked to report about their satisfaction at postoperative month 6 . The satisfaction was rated on a 3 step

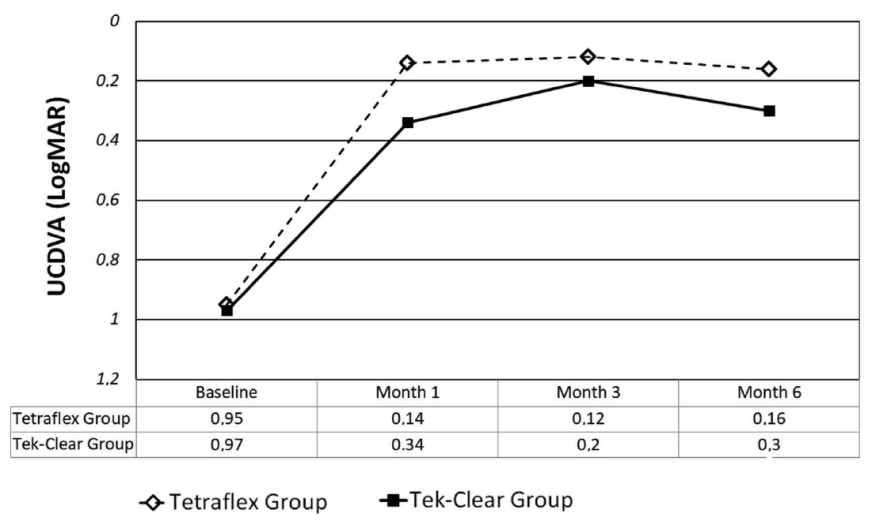

Figure 1. The uncorrected distance visual acuity levels between the two groups at different time points

UCDVA: Uncorrected distance visual acuity, LogMAR: Logarithm of the minimum angle of resolution

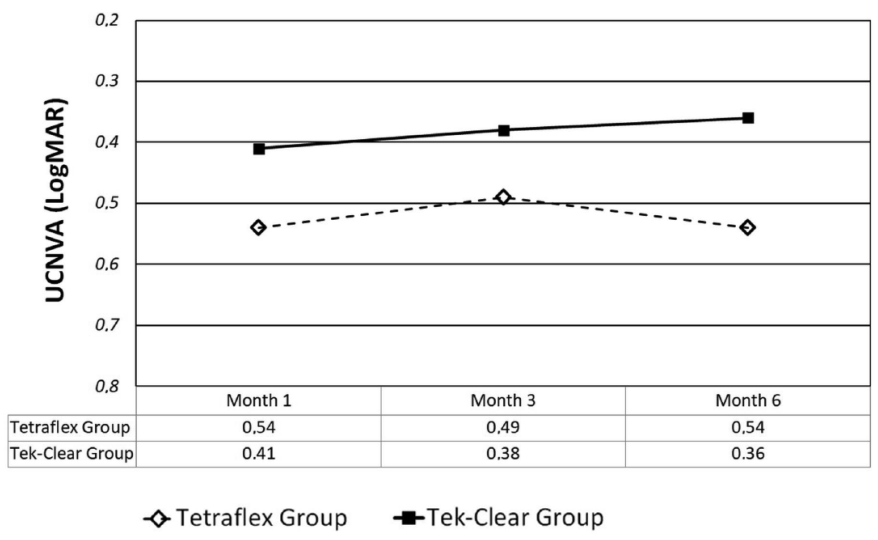

Figure 2. The uncorrected near visual acuity levels between the two groups at different time points

UCNVA: Uncorrected near visual acuity, LogMAR: Logarithm of the minimum angle of resolution 
grading; very satisfactory, intermediately satisfactory, and not satisfactory subjectively. All of the included patients answered the question as very satisfactory. Also, 10 of the 12 (83.3\%) bilaterally AIOL implanted patients obtained total spectacle independence at postoperative month 6 .

\subsection{Postoperative complications}

No postoperative complications like decentration or dislocation of the AIOLs, corneal edema, inflammation, increased IOP, cystoid macular edema was detected in any of the patients. Also, any of the patients did not complain about halo or glare during the postoperative follow-up period.

At postoperative month $6,29.4 \%$ of the patients in the Tetraflex group and $28.8 \%$ of the Tek-clear group showed mild posterior capsule opacification $(p<0.05)$. None of the patients required laser capsulotomy because posterior capsule opacification (PCO) was not clinically significant and was not obscured the visual axis in any of the groups (14).

\section{DISCUSSION}

We compared the visual and functional outcomes of 2 AlOLS in this prospective study. Both of the IOLs had single optics and worked with accommodative effort. Visual outcomes were similar between the two IOLs. Both of them showed improvement in both near and distance visual acuities. No statistically significant difference was found between them in regards to visual outcomes except postoperative uncorrected distance and near visual acuities. The patients implanted with the Tek-clear AIOL showed a myopic shift, which caused better UCDVA with Tetraflex and better UCNVA with Tek-clear at postoperative month 1,3 , and 6 . The two important outcomes

\begin{tabular}{|c|c|c|c|}
\hline & Tetraflex & Tek-clear & $\mathrm{p}$ \\
\hline \multicolumn{4}{|c|}{ SE (D) (Mean \pm SD) } \\
\hline Month 1 & $-0.43 \pm 0.68$ & $-1.32 \pm 0.57$ & $<0.0001$ \\
\hline Month 3 & $-0.29 \pm 0.74$ & $-1.24 \pm 0.59$ & $<0.0001$ \\
\hline Month 6 & $-0.18 \pm 0.78$ & $-1.27 \pm 0.55$ & $<0.0001$ \\
\hline \multicolumn{4}{|c|}{ AA (D) (Defocus method) } \\
\hline Month 3 & $-1.04 \pm 0.30$ & $-1.10 \pm 0.27$ & 0.4 \\
\hline Month 6 & $-1.15 \pm 0.27$ & $-1.17 \pm 0.29$ & 0.8 \\
\hline \multicolumn{4}{|c|}{$\triangle \mathrm{ACD}(\mathrm{mm})$} \\
\hline Month 3 & $0.32 \pm 0.16$ & $0.33 \pm 0.33$ & 0.2 \\
\hline Month 6 & $0.37 \pm 0.16$ & $0.37 \pm 0.30$ & 0.2 \\
\hline \multicolumn{4}{|c|}{$\begin{array}{l}\text { SE: Spherical equivalent, D: Diopter, AA: Accommodation amplitude, } \triangle \text { ACD: The } \\
\text { difference between before and after pilocarpine induced anterior chamber depth, } \\
\text { p: P value, SD: Standard deviation }\end{array}$} \\
\hline
\end{tabular}

of this study were that the Tek-clear group was found to be more myopic than the Tetraflex group $(-1.27 \mathrm{D}$ vs $-0.18 \mathrm{D}$ at month 6); therefore the uncorrected near vision was better in Tek-clear, and uncorrected distance vision was better with Tetraflex as expected. The significances between the uncorrected distance and near visual acuity levels were probably secondary to the postoperative myopic shift of the Tek-clear group. We supposed that this shift might be secondary to the keratometric/biometric errors or effective lens position or a constant of the IOL. All of the keratometric and biometric measurements were made by a single physician (HNT), and the anterior chamber parameters were similar between the two groups. Therefore, we ruled out the first two probable reasons for the myopic shift. Therefore, we supposed that this phenomenon was probably caused by a constant of the Tek-clear AIOL. Although the myopic shift in Tek-clear implanted eyes was reported in another study (15), we could not be sure about this, so we informed the company in this regard. The main anatomical outcomes of the study showed a similarity between the two IOLs, both subjective and objective AA outcomes were not statistically different between the two groups.

In the literature, there are several studies regarding the outcomes of single optic AIOLs $(10,16-24)$. The most evaluated one was Crystalens, which is the first Food and Drug Administration approved $\operatorname{AIOL}(7,8,19)$. In most of the reports, it was demonstrated that Crystalens showed improvement in both distance and near visual acuity $(7,8)$. In a study by Karavitaki et al. (19), the long-term visual acuity outcomes after bilateral Crystalens implantation were evaluated. They included 50 eyes of the 25 patients and reported the visual outcomes and complications after a mean follow-up period of 42 months. The mean UCDVA was found to be improved from 0.56 to 0.19 LogMAR, and BCDVA was found to increase from 0.17 to 0.05 LogMAR at the last follow up visit. The uncorrected intermediate and near visual acuity was $\geq 12$. No intra-or postoperative complications were reported, except posterior capsule opacification in 12 of the 50 eyes during the first year (19). Another widely evaluated single optic AIOL is $1 C U(5,20)$. In a randomized comparative trial by Harman et al. (20), binocular near vision performance was compared among patients who were implanted with the 1CU accommodating, multifocal, and monofocal IOL. Initially, 90 patients were included in the study, and 64 of them completed the follow-up period of 18 months. Mean uncorrected and corrected distance visual acuities were found to be increased in all of the three groups at months 3 and 18 postoperatively. The UCNVA and accommodative range were better in both $1 \mathrm{CU}$ than monofocal IOL group at month 3 
and 18 as expected (20). The Tetraflex and Tek-clear AIOLs are the two other AIOLs in the market that were evaluated in this study $(7,8)$. Wang et al. (21) evaluated the visual performance of Tetraflex in 23 eyes of 23 patients with cataract. After a mean follow-up period of 12 months, the Tetraflex implanted eyes showed significant improvement in regards to UCNVA and UNDVA. Total spectacle independence was achieved, \%34.7 of the patients. Tan et al. (23) compared the performances of accommodative, multifocal, and monofocal IOLs in a study in which Tetraflex AIOL was used. The Tetraflex group of the study showed improvement in distance and near visual acuity at the follow-up visits at postoperative months 3 and 12, and $60 \%$ of the patients achieved total spectacle independence. The percentage of achieving total spectacle independency was nearly twice when compared with the study by Wang et al. (21). This might be due to the high bilateral operation rate of the patients who were included in the study by Tan et al. (23) in contrast to the unilaterality of the patients in the study of Wang et al. (21). Also $83.3 \%$ of bilaterally AIOL implanted patients did not require spectacle correction in our study that supported this idea. In another study by Beiko (10), the visual performances of Crystalens, Tetraflex, and monovision with a monofocal IOL were compared. No statistical difference was reported in visual outcomes among the three groups. Wolffsohn et al. (24) investigated the mechanism of action of the Tetraflex IOL by assessing the objective amplitude-of-accommodation via autorefraction, anterior chamber depth, and pupil size via optical coherence tomography, and IOL flexure via aberrometry. They reported a decrease in the pupil size was, and interestingly the IOL was found in the same position with the accommodation effort; however, the optical aberrations were found to be increased. Therefore, they postulated that the accommodative benefits of Tetraflex might be due to optical aberrations. The Tek-clear AIOL was rarely studied in the literature (15). Sadoughi et al. (15) compared the distance and near visual function after cataract surgery among the patients who were implanted with Crystalens AIOL, Tek-clear AIOL, or a monofocal IOL. After a follow-up period of 6 months, distance and visual acuity levels were found to be similar among the three groups. However, near visual acuity functions were better in both of the AIOL groups than the monofocal IOL group. The spherical and cylindrical refraction error of the Tek-clear group was -0.14 and -1.0 diopters, respectively. Moreover, this was arithmetically greater than the other two groups, which were similar to the postoperative refractive error of the Tek-clear subgroup of our study. Also, the patients with postoperative ametropia $>-0.50$ diopters were excluded from the study by Sadoughi et al. (15), which probably decreased the mean postoperative myopic shift among the Tek-clear implanted eyes.

No significant postoperative complications were detected in both of the groups except PCO, which was the most important complication of AIOLs (25). It was thought to be a drawback of the AIOLs because the anteroposterior movement of AIOLs was proposed to induce early PCO in the AIOL implanted eyes; also the probable change in anterior segment parameters after YAG laser capsulotomy was another hesitation about the AIOLs $(25,26)$. However, the rate of PCO in AIOLs was reported to be similar to other hydrophilic IOLs, and the accommodative ability was reported to be unchanged after YAG laser capsulotomy. The PCO rate was similar between the Tetraflex and Tek-clear groups in our study, and none of the patients required YAG laser capsulotomy during the follow-up period of the study.

The main limitation of the study was the small number of included patients and non-randomized study design. Also, the choice of AIOL did not depend on any special consideration because we had the previously bought AIOLs in our institution and had to implant the AIOLs according to the biometrydefined power of the AIOL. However, we compared the visual and accommodative outcomes of these two different AIOLs prospectively in the literature for the first time in patients with cataracts and obtained some positive results, which were discussed in detail.

\section{CONCLUSION}

In conclusion, both AIOLs used in our group of patients showed superior performances in regards to visual outcomes. The only difference was found in the postoperative uncorrected distance and near visual acuity levels and manifest refraction. The Tekclear group showed a myopic shift postoperatively, which caused the group to demonstrate a better outcome in uncorrected near vision. In contrast, the Tetraflex group showed a better outcome concerning uncorrected distance vision. Therefore, we might propose some recommendations according to the results of this study. If a patient requires a perfect distance visual acuity and a good or intermediate near visual acuity, then Tetraflex IOL might be the better choice. In contrast, if a patient requires a very good near visual acuity and a good or intermediate distance visual acuity, then Tek-clear IOL might be the better choice. Of course, this was a pilot study conducted with a relatively low number of patients, and we might only assume these results and recommendations. Further prospective, randomized, comparative studies are needed to clarify our results. 


\section{Ethics}

Ethics Committee Approval: Ethics committee approval was obtained from the Ethics Committee of Bakırköy Dr. Sadi Konuk Training and Research Hospital (2011/117).

Informed Consent: Written informed consent was obtained from the patients.

Peer-review: Externally and internally peer-reviewed.

\section{Authorship Contributions}

Concept: H.N.T., U.Y., I.U.O., A.Ö., Design: H.N.T., U.Y., I.U.O., A.Ö., Data Collection or Processing: H.N.T., U.Y., I.U.O., Analysis or Interpretation: H.N.T., U.Y., I.U.O., A.Ö., Literature Search: H.N.T., A.Ö., Writing: H.N.T., A.Ö.

Conflict of Interest: No conflict of interest was declared by the authors.

Financial Disclosure: The authors declared that this study received no financial support.

\section{REFERENCES}

1. Doane JF, Jackson RT. Accommodative intraocular lenses: considerations on use, function and design. Curr Opin Ophthalmol 2007;18:318-24.

2. Martin H, Guthoff R, Terwee T, Schmitz KP. Comparision of the accomodation theories of Coleman and of Helmholtz by finite element simulations. Vis Research 2005;45:2910-5.

3. Coleman J, Fish S. Presbyopia, accomodation, and the mature catenary. Ophtalmol 2001;108:1544-51.

4. Ostrin LA, Glasser A. Accommodation measurements in a prepresbyopic and presbyopic population. J Cataract Refract Surg 2004;30:1435-44.

5. Papadopoulos PA, Papadopoulos AP. Current management of presbyopia. Middle East Afr J Ophthalmol 2014;21:10-7.

6. Greenwood M, Bafna S, Thompson V. Surgical Correction of Presbyopia: Lenticular, Corneal, and Scleral Approaches. Int Ophthalmol Clin 2016;56:149-66.

7. Alió JL, Alió Del Barrio JL, Vega-Estrada A. Accommodative intraocular lenses: where are we and where we are going. Eye Vis (Lond) 2017;4:16.

8. Pepose JS, Burke J, Qazi M. Accommodating Intraocular Lenses. Asia Pac J Ophthalmol (Phila) 2017;6:350-7.

9. Alio JL, Plaza-Puche AB, Férnandez-Buenaga R, Pikkel J, Maldonado M. Multifocal intraocular lenses: An overview. Surv Ophthalmol 2017;62:611-34.

10. Beiko GH. Comparison of visual results with accommodating intraocular lenses versus mini-monovision with a monofocal intraocular lens. J Cataract Refract Surg 2013;39:48-55.

11. Eğrilmez S, Eğrilmez ED, Akkın C, Kaskaloglu M, Yagcı A. Uluslararası standartlara uygun bir Türkçe yakın okuma eșeli. T Oft Gaz 2004;34:40412.
12. Nemeth G, Lipecz A, Szalai E, Berta A, Modis L Jr. Accommodation in phakic and pseudophakic eyes measured with subjective and objective methods. J Cataract Refract Surg 2013;39:1534-42.

13. Leydolt C, Menapace R, Stifter EM, Prinz A, Neumayer T. Effect of primary posterior continuous curvilinear capsulorrhexis with posterior optic buttonholing on pilocapine-induced IOL shift. J Cataract Refract Surg 2012;38:1895-901.

14. Raj SM, Vasavada AR, Johar K, Vasavada VA, Vasavada VA. Post-Operative Capsular Opacification: A Review. Int J Biomed Sci 2007;3:237-50.

15. Sadoughi MM, Einnollahi B, Roshandel D, Sarimohammadli M, Feizi S. Visual and refractive outcomes of phacoemulsification with implantation of accommodating versus standard monofocal intraocular lenses. J Ophthalmic Vis Res 2015;10:370-4.

16. Zare Mehrjerdi MA, Mohebbi M, Zandian M. Review of Static Approaches to Surgical Correction of Presbyopia. J Ophthalmic Vis Res 2017;12:4138.

17. Tomás-Juan J, Murueta-Goyena Larrañaga A. Axial movement of the dual-optic accommodating intraocular lens for the correction of the presbyopia: optical performance and clinical outcomes. J Optom 2015;8:67-76

18. Lindstrom RL, Macrae SM, Pepose JS, Hoopes PC Sr. Corneal inlays for presbyopia correction. Curr Opin Ophthalmol 2013;24:281-7.

19. Karavitaki AE, Pallikaris IG, Panagopoulou SI, Kounis GA, Kontadakis G, Kymionis GD. Long-term visual outcomes after Crystalens $\left.{ }^{\circledR}\right)$ HD intraocular lens implantation. Clin Ophthalmol 2014;8:937-43.

20. Harman FE, Maling S, Kampougeris G, Langan L, Khan I, Lee N, et al. Comparing the $1 \mathrm{CU}$ accommodative, multifocal, and monofocal intraocular lenses: a randomized trial. Ophthalmology 2008;115:9931001.

21. Wang JW, Sun K, Su YD, Liu X, Du Y, Zhong ZW. Long-term clinical outcomes after implantation of Tetraflex accommodative intraocular lens. Int Eye Sci 2013;13:225-8.

22. Dong Z, Wang NL, Li JH. Vision, subjective accommodation and lens mobility after TetraFlex accommodative intraocular lens implantation. Chin Med J (Engl) 2010;123:2221-4.

23. Tan N, Zheng D, Ye J. Comparison of visual performance after implantation of 3 types of intraocular lenses: accommodative, multifocal, and monofocal. Eur J Ophthalmol 2014;24:693-8.

24. Wolffsohn JS, Davies LN, Gupta N, Naroo SA, Gibson GA, Mihashi T, Shah S. Mechanism of action of the tetraflex accommodative intraocular lens. J Refract Surg 2010;26:858-62.

25. Takakura A, Iyer P, Adams JR, Pepin SM. Functional assessment of accommodating intraocular lenses versus monofocal intraocular lenses in cataract surgery: metaanalysis. Journal of Cataract and Refractive Surgery 2010;36:380-8.

26. Nguyen NX, Seitz B, Reese S, Langenbucher A, Küchle M. Accommodation after $\mathrm{Nd}$ : YAG capsulotomy in patients with accommodative posterior chamber lens 1CU. Graefes Arch Clin Exp Ophthalmol 2005;243:120-6. 